\title{
Application of mesocolon rotation and reverse puncture in total laparoscopic sigmoid vaginoplasty
}

\author{
WENBIN ZHANG ${ }^{1 *}$, CHUNXING LI $^{1 *}$, WENJIE CHENG ${ }^{2}$, TAO LI ${ }^{1}$, \\ HUA GAO $^{1}$, HUIHUI ZHANG ${ }^{3}$ and XINCAI XU ${ }^{1}$ \\ ${ }^{1}$ Department of Gastrointestinal Tumors, The First Affiliated Hospital of Xinjiang Medical University, \\ Urumqi, Xinjiang 830054; ${ }^{2}$ Department of General Surgery, Luopu County People's Hospital, Hotan, \\ Xinjiang 848000; ${ }^{3}$ The 12th Division Hospital of Xinjiang Production and Construction Corps, \\ Urumqi, Xinjiang 830013, P.R. China
}

Received April 27, 2018; Accepted May 31, 2019

DOI: $10.3892 /$ etm.2019.7920

\begin{abstract}
The aim of the present study was to explore the clinical efficacy of mesocolon rotation and reverse puncture in total laparoscopic sigmoid vaginoplasty (LSV). The clinical data of 11 patients (unmarried, $n=4$; married, $\mathrm{n}=7$ ) with congenital absence of a vagina who underwent treatment of total laparoscopic sigmoid vaginoplasty at the First Affiliated Hospital of Xinjiang Medical University (Urumqi, China) between March 2013 and March 2016 were retrospectively analyzed. In all patients, the surgical method included sigmoid mesocolon rotation and reverse puncture. The vaginal depth, the first sexual intercourse time and female sexual function indexes were recorded. The average operation time was $187 \pm 19 \mathrm{~min}$, the average intra-operative blood loss was $132 \pm 24 \mathrm{ml}$, the time to the first meal after surgery was $4.3 \pm 1.1$ days, the average post-operative hospital stay was $7.5 \pm 1.2$ days, the post-operative short-term complication rate was $36.3 \%$ and the time to the first sexual intercourse was $3.0 \pm 0.3$ months. The post-operative follow-up results indicated that the anatomical standard was reached in all of the 11 patients. Among the seven married patients, five patients were satisfied with their sex lives after the operation. In terms of psychosexual desire, only one married patient felt no sexual arousal. The other married patients had good sexual relations, function and satisfaction. In conclusion, the application of mesocolon rotation and reverse puncture in total LSV is safe and feasible.
\end{abstract}

Correspondence to: Dr Xincai Xu, Department of Gastrointestinal Tumors, The First Affiliated Hospital of Xinjiang Medical University, 137 South Road of Carp Mountain, Urumqi, Xinjiang 830054, P.R. China

E-mail: xia51195113@163.com

*Contributed equally

Key words: congenital absence of a vagina, total laparoscopic sigmoid vaginoplasty, mesocolon, sigmoid colon

\section{Introduction}

The most common type of congenital absence of a vagina is Mayer-Rokitansky-Kuster-Hauser (MRKH) syndrome. At present, vaginoplasty is the best method for the treatment of MRKH syndrome (1). Since the first development of vaginoplasty, $>100$ other surgical methods have been developed. The major difference between the various methods is the tissue material used to create the artificial vagina. The purpose of all operations is to make the anatomy and function of the artificial vagina as close as possible to those of a normal vagina $(2,3)$. The replacement of traditional open surgery with a simple operation (i.e., laparoscopy) and the use of an autograft substitute as an allograft are currently major development trends in vaginoplasty (4).

Intestinal vaginoplasty is widely performed in the clinic, as the resulting artificial vagina has a function closer to that of a normal vagina, natural lubrication and early intercourse function $(5,6)$. Compared with the small intestine, the sigmoid colon has the advantages of tolerance to trauma due to its thickness, no requirement of wearing a mold to maintain the operation effect for a long time, moderate secretion and capacious internal cavity $(7,8)$. With the introduction of fine laparoscopic instruments, sigmoid colon vaginoplasty has gradually become a completely 'minimally invasive' laparoscopic procedure. In recent years, patients' demand for improved post-operative physiological and psychological quality has increased. As a result, surgeons must now consider how best to optimize laparoscopic surgical procedures to respond to this demand while reducing post-operative complications. Improvements in the operative procedure may enhance the quality of laparoscopic surgery.

Between March 2013 and March 2016, total laparoscopic sigmoid vaginoplasty (LSV) was performed on 11 patients at our institution. All of the surgeries were performed with sigmoid mesocolon rotation and reverse puncture under laparoscopy. In the present study, the clinical data of these patients were retrospectively analyzed to explore the clinical effect of total LSV, and data regarding the procedures and outcomes were reported. The clinical effect of LSV was compared to laparoscopic sigmoid colovaginoplasty, reported 
in a previous study (9), by comparing the female sexual function indexes.

\section{Patients and methods}

Patients. The study comprised 11 patients (married, $\mathrm{n}=7$; unmarried, $n=4$ ) diagnosed with MRKH syndrome and had primary amenorrhea prior to the operation. The average age of the patients was $22.8 \pm 2.7$ years (ranged from 18 to 27 years) and the body mass index was $24.9 \pm 1.9 \mathrm{~kg} / \mathrm{m}^{2}$. None of the patients had a history of diseases associated with MRKH syndrome or a familial history of MRKH syndrome. One of the patients had previously undergone laparoscopic appendectomy and two patients had undergone laparoscopic cholecystectomy. None of these patients experienced any complications after the operation. Urinary system B-ultrasound indicated no obvious abnormalities in the bilateral kidneys, bilateral ureter and bladder. Uterine imaging and sex hormone levels were normal in all patients. All of the patients had normal external female genitalia and normal ovarian function. The chromosomal pattern was $46, \mathrm{XX}$. In line with standard procedures, enteroscopy was performed prior to the operation, revealing no obvious abnormalities. All of the patients had requested total LSV after being informed of various vaginal shaping methods.

Bowel preparation. All of the patients consumed a semiliquid diet for 3 days prior to the operation. Metronidazole was also prescribed. At $12 \mathrm{~h}$ prior to the operation, the patients received a cleansing enema and received intravenous nutrition. Prophylactic cephalosporin antibiotics were administered 30 min prior to the operation. Traditional skin preservation of the perineal region was performed prior to the operation.

Pre-operative preparation. After receiving a general anesthetic, the patients were placed in the lithotomy position (left high right low, Trendelenburg position). In the middle of the umbilicus, a veress needle was inserted to establish a pneumoperitoneum and maintain a pressure of $14 \mathrm{mmHg}$. A trocar was then placed in site, which is the same procedure as during colorectal surgery. The ureters and ovaries in the abdominal cavity were then examined and the depth of the pelvic cavity was measured. The length of the sigmoid colon and mesocolon were assessed. The distribution and shape of the arterial branches were observed by using transillumination of the mesocolon to determine the length of the sigmoid colon and artery revascularization.

Operative method. The operation was divided into 7 steps. The first step was to probe the pelvic cavity and construct a tunnel. Under the laparoscopy, the pelvic floor peritoneum was cut open and the rectovesical pouch peritoneum was dissected. The pre-rectum space was then separated by using sharp and blunt dissection, and the width was $5-6 \mathrm{~cm}$. The vagina and abdomen were then examined and a tunnel linking the external vaginal stump was constructed. The second step was to separate the sigmoid colon side peritoneum. The third step was to locate the distal end of the transplanted intestinal segment. The height of the sacral promontory was considered the distal end of the transplanted intestinal segment (AB terminus; Fig. 1), and was divided under laparoscopy. The fourth step was to locate the proximal end of the transplanted intestinal segment and to free it completely. According to the depth of the pelvic cavity $+1 / 2$ length of the mesocolon or a normal vaginal length $+5 \mathrm{~cm}$, the length of the transplanted intestinal segment was $16-18 \mathrm{~cm}$ due to keeping the blood supply and meeting the length requirement for transplantation. The length of a normal vagina is $13-14 \mathrm{~cm}$, with an average length of $12.8 \mathrm{~cm}$. The measurement was performed from the distal end of the transplanted intestinal segment (AB terminus) using a scale line prepared prior to the operation. The proximal segment of the sigmoid colon at a distance of $18 \mathrm{~cm}$ from the $\mathrm{AB}$ end served as the proximal end of the transplanted intestinal segment (CD terminus). The branches and shape of the sigmoid colon were then observed, and the anterior and posterior peritoneum of the mesocolon were freed along the $\mathrm{AB}$ and $\mathrm{CD}$ dotted line (Fig. 1). Subsequently, the lower artery of the sigmoid colon and the descending branch of the left colon were occluded and the main sigmoid colon artery was retained. The fifth step was to cut the transplanted intestinal segment under the laparoscopy and complete the anastomosis between the rectum and sigmoid colon. The nail seat anastomat (PROXIMATE LLS Curved and Straight Intraluminal Staplers; Johnson \& Johnson) was introduced into the pelvic cavity from the tunnel. The sigmoid colon tube was longitudinally split $2 \mathrm{~cm}$ at $1.5 \mathrm{~cm}$ on the CD terminus of the transplanted intestinal segment, and the anastomat was placed in the intestinal tube through this incision and moved to the upper section of the CD terminus. The puncture outfit of the nail seat went out of the intestines from $1.0 \mathrm{~cm}$ above the $\mathrm{CD}$ terminus. The proximal end of the sigmoid colon was then removed using a closed cutter and the intestinal segment to be transplanted was completely dissociated. Next, a stapler was inserted into the rectum through the anus to the broken end, and the proximal end of the sigmoid colon was anastomosed to the end of the rectum. The sixth step was to rotate the intestinal segment mesocolon to be transplanted 180 degrees clockwise horizontally to the pelvic cavity. The seventh step was vaginoplasty (8).

Postoperative treatment. After the operation, the artificial vagina did not require a vaginal mold and was washed once with clear water every other day. Routine post-operative care included flushing around the anus twice a day. Prior to the recovery of intestinal function, the patients received intravenous nutrition support. After anal exhaust, the diet of the patients gradually transitioned from a liquid to a semi-liquid diet. All of the patients were given prophylactic antibiotics and any changes in abdominal signs were closely observed.

Follow-up. For the first month after the operation, the patients were followed up once per week by using questionnaire as previous (10) and telephone interview. Two months after the operation, the period of follow-up was once every 2 weeks. Three years after the operation, the period of follow-up was once every 3 months. The follow-up included assessments of vaginal size, volume, length and color, as well as vaginal discharge and secretions. The married patients were also questioned about aspects of their sex lives.

Control group of laparoscopic sigmoid colovaginoplasty. After selecting a sigmoid segment based on vascular anatomy 


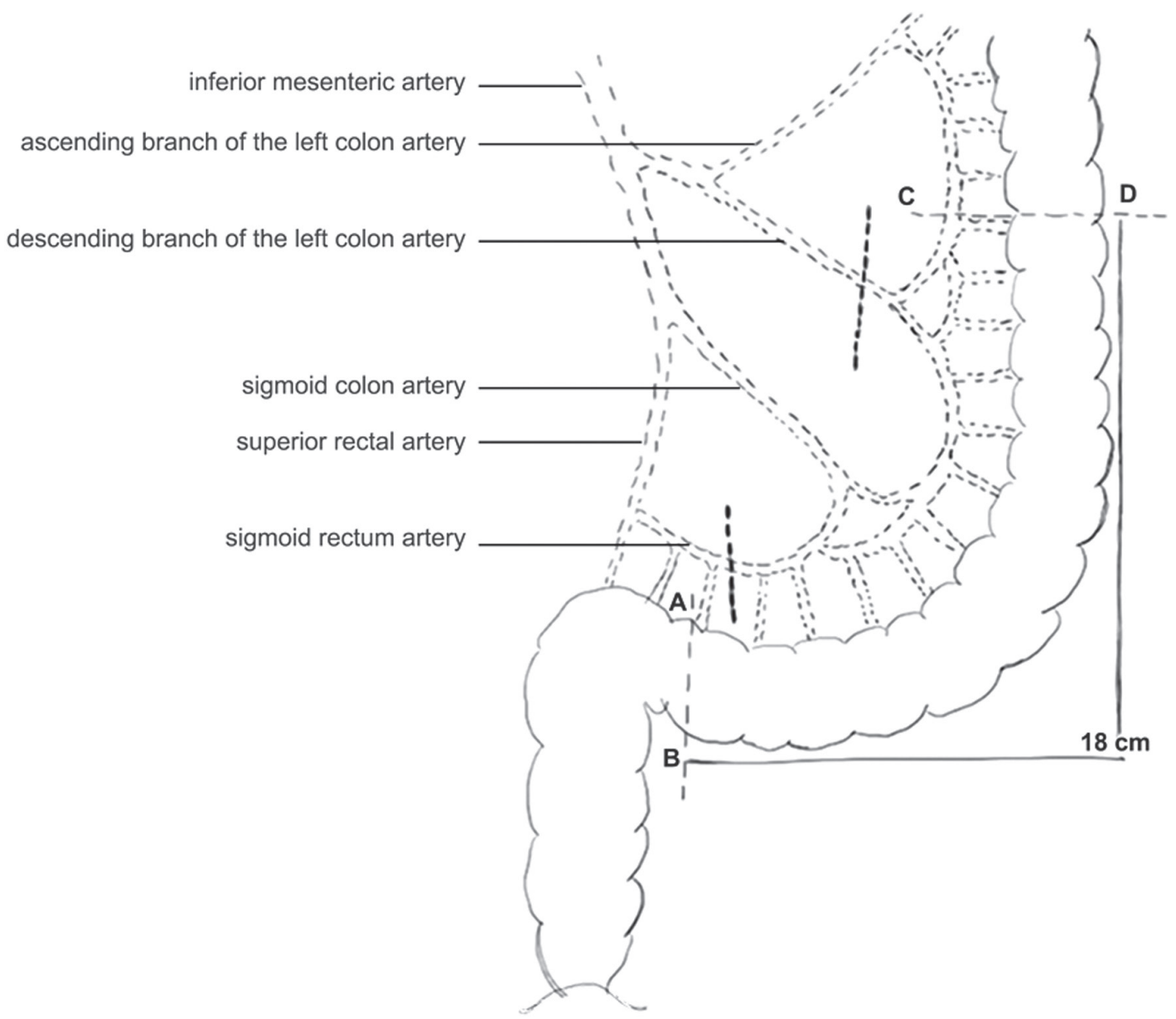

Figure 1. Schematic depicting the supply of the sigmoid colon artery and the location of the transplanted intestinal segment. The main sigmoid colon artery was retained.

and mesenteric length, the segment was mobilized using a Harmonic scalpel. Through a 3 -cm Pfannenstiel incision, the dissociated sigmoid colon was pulled out to exteriorize and create a purse. Continuity of the intestinal tract was restored using a circular mechanical suture through the rectum. At the tip of the newly created perineal space, an incision was made using a Harmonic knife; the size of the incision was sufficient to allow free passage of the isolated sigmoid colon segment. Subsequently, the sigmoid autograft was sutured to achieve construction of a normal-appearing vulva.

Statistical analysis. SPSS 22.0 software (IBM Corp.) was used to perform the statistical analysis. Values are expressed as the mean \pm standard deviation or $n(\%)$. Statistical analyses were performed using a Student's t-test for measurement data and a $\chi^{2}$ test for count data. $\mathrm{P}<0.05$ was considered to indicate statistical significance.

\section{Results}

Perioperative data. As presented in Table I, the average operation time was $187 \pm 19 \mathrm{~min}$, the average intra-operative blood loss was $132 \pm 24 \mathrm{ml}$, the time of the first meal after surgery was $4.3 \pm 1.1$ days and the average post-operative hospital stay was $7.5 \pm 1.2$ days. The post-operative short-term complication rate was $36.3 \%$ and the time of the first sexual intercourse was $3.0 \pm 0.3$ months. The operation time, intraoperative blood loss, time of first meal after surgery, vaginal depth and first sexual intercourse time of those who received total LSV were significantly better compared with the control group of laparoscopic sigmoid colovaginoplasty, whilst LSV also had higher complication rate than laparoscopic sigmoid colovaginoplasty (Table I). At two weeks after the surgery, the abdominal laparoscopic incision had healed well. Four cases developed surgical complications, such as excessive vaginal secretion, vaginal opening stenosis and intermittent dull pain and discomfort. The vaginal mucosa was pink with a small amount of mucus.

Postoperative vaginal recovery during 3 year follow up. Anatomical success was defined as the ability to insert two fingers with ease into the vagina and a vaginal length of $>6 \mathrm{~cm}$ (11). The follow-up results at 3 years after the operation indicated that the depth and width of the newly created vagina in all of the 11 patients reached the anatomical standard, including vaginal secretions and the possibility of sexual intercourse $(12,13)$. The average artificial vagina depth was $16.4 \pm 0.6 \mathrm{~cm}$ (Table I).

Female sexual function index (FSFI). Among the 7 married patients, 5 patients were satisfied with their sex lives after the operation. In one patient, vaginal secretion was excessive for the first 6 months. One married patient felt no sexual arousal. 
The other married patients had active sexual relations, function and satisfaction. The FSFIs of the 7 married patients who received total LSV were not significantly different from those in the control group of laparoscopic sigmoid colovaginoplasty (Table II) (9). All subjects, including the control group, were assessed in an identical/comparable way.

\section{Discussion}

With the development of minimally invasive surgery, the goal of surgeons is to improve the post-operative psychological and physiological integrity of patients with congenital absence of a vagina (14). This goal may be achieved by non-invasive laparoscopy using fine laparoscopic instruments. However, performing total LSV is relatively complex, as the procedure involves resection of the transplanted intestinal segment, the placement and fixation of a nail seat anastomat and anastomosis of the broken ends of the intestine under laparoscopy. Additional issues for surgeons are to ensure a sufficient length of the sigmoid colonic flap and vascular bundles, and adequate blood supply has always been a challenge for surgeons $(15,16)$. Therefore, in addition to improving operative details, it is necessary to fully understand the mesenteric vascular anatomy, which is important for increasing the quality of laparoscopic surgery.

A key issue in LSV is dealing with the problem of the large tension in down-placing the transplanted intestinal segment. The two major factors that affect the success of down-placement of the transplanted intestinal segment are the mesocolon and the vascular distribution of the mesocolon. The sigmoid colon arteriovenous vein is an important branch of the inferior mesenteric artery and vein. Its position is closer to the pelvic cavity as compared with the ascending branch and descending branches of the left colon artery (17), which is the most important branch of the transplanted intestinal segment. In the cases described in the present study, the sigmoid colon was rotated 180 degrees clockwise to the pelvic cavity, and vaginoplasty was then performed. This procedure is relatively simple, and it does not necessitate excessive dissociation of the sigmoid mesocolon and blood vessels. It also minimizes the destruction of the vascular arch and ensures the blood supply of the transplanted intestinal segments. Mesocolon rotation has two major advantages: First, the arteriovenous location of the sigmoid colon is close to the pelvic cavity, which is near the surgical site; second, the activity of the sigmoid mesocolon is high. Thus, the transplanted intestinal segment may be freed relatively easily, followed by down-placement in the pelvic cavity and pulling it to the vaginal entrance. The influence of the mesocolon and vascular distribution on mesocolon rotation is relatively small. Thus, mesocolon rotation has a wide range of applications. As compared with the traditional longitudinal down-placement of the transplanted intestinal segment, mesocolon rotation is superior in terms of solving the problem of large tension in down-placing the transplanted intestinal segment. A problem with longitudinal down-placement of the transplanted intestinal segment is that if the sigmoid mesocolon is too short or there is adhesion in the intestinal tube and pelvic cavity during the operation, excessive traction of the transplanted intestinal segment may cause ischemic tissue necrosis. 
Table II. Comparison of the female sexual function indexes of the married patients between total LSV and laparoscopic sigmoid colovaginoplasty.

\begin{tabular}{lccc}
\hline Index & Total LSV (married patients, $n=7)$ & ${\text { Laparoscopic sigmoid colovaginoplasty }(\mathrm{n}=14)^{\mathrm{a}}}^{\mathrm{P}-\mathrm{value}}$ \\
\hline Sexual desire & $3.2 \pm 0.5$ & $4.19 \pm 0.24$ & 0.241 \\
Sexual arousal & $3.6 \pm 1.2$ & $4.19 \pm 0.07$ & 0.672 \\
Lubrication & $2.4 \pm 1.1$ & $4.19 \pm 0.07$ & 0.216 \\
Orgasm & $4.6 \pm 1.5$ & $4.07 \pm 0.32$ & 0.304 \\
Satisfaction & $3.3 \pm 1.5$ & $4.41 \pm 0.25$ & 0.215 \\
Pain & $3.1 \pm 2.1$ & $4.22 \pm 0.33$ & 0.402 \\
Total points & $20.2 \pm 7.9$ & $24.79 \pm 1.17$ & 0.202 \\
\hline
\end{tabular}

${ }^{a}$ Cases from Cao et al (9). The same questionnaire and interview system was used as for Cao et al. LSV, laparoscopic sigmoid vaginoplasty.

To solve the problem of large tension when down-placing the transplanted intestinal segment, Karateke et al (18) suggested the following: First, in cases where the vascular distribution is narrow but the mesocolon is long, all of the sigmoid colon artery branches may be retained to create a vagina directly or after rotating by 180 degrees. Second, in cases where the mesocolon is relatively short but the sigmoid colon artery has wide branches and a large area, it may cut off the proximal end and middle part of the mesocolon may be cut off, followed by rotation of the intestinal segment by 180 degrees clockwise without affecting the blood supply. In addition, the length of the mesocolon may be extended by transversely cutting the anterior and posterior peritoneum in the root or middle part of the mesocolon, thereby reducing the tension (19). Due to the unique anatomical structure of the sigmoid colon, mesocolon rotation may be employed safely and effectively to solve the problem of tension while down-placing the transplanted intestinal segment.

In the present study, the post-operative follow-up indicated that all of the 11 patients achieved normal anatomical function. Among the 7 married patients, 5 patients were satisfied with their sex lives after the operation, and 1 patient had vaginal pain or discomfort during sex. One married patient felt no sexual arousal during intercourse. The other married patients had active functioning sexual relations. The FSFIs of the 7 married patients who received total LSV were not significantly different from those in the control group of laparoscopic sigmoid colovaginoplasty. Overall, the FSFI indicated a high degree of sexual satisfaction after total LSV. Although post-operative intestinal complications, including vaginal contracture, excessive vaginal secretion and vaginal stenosis may occur (20), these implications may still be effectively controlled and treated.

The limitations of the present study are the short follow-up times for the patients ( $<3$ years). Further long-term follow-up studies are therefore required. In addition, it may be recommended to perform left colon angiography prior to the surgery, but this was not performed in the present study.

In summary, the application of mesocolon rotation and reverse puncture in total LSV is safe and feasible. As compared with traditional surgery, the operation time was short, the wound was small and the operative procedures affected by the mesocolon and vascular distribution was less. To improve long-term outcomes and ensure the best surgical results, multidisciplinary teams and comprehensive psychological and medical support are required. The sample size in the present study was small and the results require to be confirmed in a large randomized multicenter study.

\section{Acknowledgements}

Not applicable.

\section{Funding}

This study was supported by Natural Science Foundation of Xinjiang Uygur Autonomous Region (grant no. 2017D01C305).

\section{Availability of data and materials}

The datasets generated and/or analyzed during the current study are available from the corresponding author on reasonable request.

\section{Authors' contributions}

WZ and CL analyzed and interpreted the patient data. WC, TL and HG collected the patients' information. XX designed the study, interpreted the data and was a major contributer in writing the manuscript. HZ interpreted the patient data, contributed significantly to drafting the study, made revisions for important intellectual content and gave final approval of the version to be published. All authors read and approved the final manuscript.

\section{Ethics approval and consent to participate}

The protocol of the present study was approved by the Ethics Committee of the First Affiliated Hospital of Xinjiang Medical University (Urumqi, China; no. K201301-01) and informed consent was obtained from all participants.

\section{Patient consent for publication}

Not applicable. 


\section{Competing interests}

The authors declare that they have no competing interests.

\section{References}

1. Creatsas G and Deligeoroglou E: Creatsas modification of Williams vaginoplasty for reconstruction of the vaginal aplasia in Mayer-Rokitansky-Kuster-Hauser syndrome cases. Womens Health (Lond) 6: 367-375, 2010.

2. Kuessel L, Wenzl R, Marschalek ML, Slavka G, Doerfler D and Husslein H: Using the Wharton-Sheares-George method to create a neovagina in patients with Mayer-Rokitansky-Küster-Hauser syndrome: A step-by-step video tutorial. Fertil Steril 106: e20-e21, 2016.

3. Krege S, Walz KH, Hauffa BP, Körner I and Rübben H: Long-term follow-up of female patients with congenital adrenal hyperplasia from 21-hydroxylase deficiency, with special emphasis on the results of vaginoplasty. BJU Int 86: 253-259, 2000.

4. Zhao YZ, Jiang H, Liu AT, Jiang DZ, Zhu XH, Qiu M,Zheng XM, Lin ZH, Yuan XB and Zhang JL: Laparoscope-assisted creation of a neovagina using pedicled ileum segment transfer. World J Surg 35: 2315-2322, 2011.

5. Zhong CX, Wu JX, Liang JX and Wu QH: Laparoscopic and gasless laparoscopic sigmoid colon vaginoplasty in women with vaginal agenesis. Chin Med J (Engl) 125: 203-208, 2012.

6. Atallah D, Salameh C, Sarkis R, Ghossain M, Safi J, Moubarak M, Ghanameh W, Moukarzel M and El Kassis N: Laparoscopic treatment of vaginal agenesis: Three cases. J Med Liban 62 227-231, 2014 (In French).

7. Ichihara K and Masumori N: Sex reassignment surgery with laparoscopic sigmoid colon vaginoplasty in a male to female transsexual: A case report. Nihon Hinyokika Gakkai Zasshi 107: 126-128, 2016 (In Japanese).

8. Bouman MB, Buncamper ME, van der Sluis WB and Meijerink WJ: Total laparoscopic sigmoid vaginoplasty. Fertil Steril 106: e22-e23, 2016.

9. Cao L, Wang Y, Li Y and $\mathrm{Xu} \mathrm{H}$ : Prospective randomized comparison of laparoscopic peritoneal vaginoplasty with laparoscopic sigmoid vaginoplasty for treating congenital vaginal agenesis. Int Urogynecol J 24: 1173-1179, 2013.

10. Meston CM: Validation of the female sexual function index (FSFI) in women with female orgasmic disorder and in women with hypoactive sexual desire disorder. J Sex Marital Ther 29: $39-46,2003$
11. Fedele L, Bianchi S, Zanconato G and Raffaelli R: Laparoscopic creation of a neovagina in patients with Rokitansky syndrome: Analysis of 52 cases. Fertil Steril 74: 384-389, 2000

12. Zhou JH, Sun J, Yang CB, Xie ZW, Shao WQ and Jin HM: Long-term outcomes of transvestibular vaginoplasty with pelvic peritoneum in 182 patients with Rokitansky's syndrome. Fertil Steril 94: 2281-2285, 2010.

13. Allen LM, Lucco KL, Brown CM, Spitzer RF and Kives S Psychosexual and functional outcomes after creation of a neovagina with laparoscopic Davydov in patients with vaginal agenesis. Fertil Steril 94: 2272-2276, 2010.

14. Karateke A, Haliloglu B, Parlak O, Cam C and Coksuer H: Intestinal vaginoplasty: Seven years' experience of a tertiary center. Fertil Steril 94: 2312-2315, 2010.

15. Zhang M, Li S, Huang X, Du H, Wang C, Zhang L, Li Y, Zhang J and Wang Z: Transumbilical single-incision laparoscopic vaginoplasty hybrid transperineal approach using a sigmoid colon segment: Initial twenty-five cases. Int Urol Nephrol 48: 1401-1416, 2016

16. Cai B, Zhang JR, Xi XW, Yan Q and Wan XP: Laparoscopically assisted sigmoid colon vaginoplasty in women with Mayer-Rokitansky-Kuster-Hauser syndrome: Feasibility and short-term results. BJOG 114: 1486-1492, 2007.

17. Grimsby GM and Baker LA: The use of autologous buccal mucosa grafts in vaginal reconstruction. Curr Urol Rep 15: 428, 2014.

18. Karateke A, Gurbuz A, Haliloglu B, Kabaca C and Koksal N: Intestinal vaginoplasty: Is it optimal treatment of vaginal agenesis? A pilot study. Surgical method of sigmoid colon vaginoplasty in vaginal agenesis. Int Urogynecol J Pelvic Floor Dysfunct 17: 40-45, 2006.

19. Pan P: Surgical and functional outcome of sigmoid colon-vaginoplasty in Mayer-Rokitansky-Kuster-Hauser syndrome. IJROG 6: 4441, 2017.

20. Kapoor R, Sharma DK, Singh KJ, Suri A, Singh P, Chaudhary H, Dubey D and Mandhani A: Sigmoid vaginoplasty: Long-term results. Urology 67: 1212-1215, 2006. 